\title{
Original
}

\section{Encuesta nacional sobre las Unidades de Cirugía Pancreática}

\author{
José Manuel Ramia a,*, Alejandro Serrablo ${ }^{b, c}$, Miguel Angel Gomez Bravo ${ }^{\text {d,e }}$ \\ y Grupo Español de Cirugía Pancreática (AEC/CE-IHPBA) ${ }^{\diamond}$
}

${ }^{a}$ Unidad de Cirugía Hepatobiliopancreática, Servicio de Cirugía General y Aparato Digestivo, Hospital Universitario de Guadalajara, Universidad de Alcalá, Guadalajara, España

${ }^{\mathrm{b}}$ Unidad de Cirugía Hepatobiliopancreática, Servicio de Cirugía General y Aparato Digestivo, Hospital Miguel Servet, Zaragoza, España

${ }^{c}$ Capítulo Español de la Asociación Internacional Hepatopancreatobiliar (IHPBA)

${ }^{\mathrm{d}}$ Unidad de Cirugía Hepatobiliopancreática, Servicio de Cirugía General y Aparato Digestivo, Hospital Virgen del Rocío, Sevilla, España

e Sección Hepatobiliopancreática (HPB) de la Asociación Española de Cirujanos

\section{INFORMACIÓN DEL ARTÍCULO}

Historia del artículo:

Recibido el 13 de enero de 2019

Aceptado el 19 de febrero de 2019

On-line el $\mathrm{xxx}$

\section{Palabras clave:}

Encuesta

Páncreas

Técnica

Cirugía

Revisión

\begin{abstract}
R E S U M E N
Introducción: Las características técnicas, humanas, científicas y asistenciales de las Unidades que atienden una patología compleja son poco estudiadas y conocidas.

Métodos: Estudio descriptivo multiinstitucional (encuesta) desarrollado conjuntamente por la sección Hepatobiliopancreática de la Asociación Española de Cirujanos y el capítulo español de la IHPBA (Asociación Internacional Hepatopancreatobiliar) sobre las características de las Unidades donde se realiza cirugía pancreática en España.

Resultados: Se enviaron 82 encuestas. Respondieron 69 centros (84\%) pertenecientes a 16 comunidades autónomas. La suma de habitantes de las áreas propias fue 23.183.262. El número medio de camas por hospital fue 673. La Unidad que realiza la cirugía pancreática es la Unidad de Cirugía Hepatobiliopancreática o Cirugía HPB y Trasplante Hepático en 56 hospitales (77\%). El número medio de cirujanos es 4,5 por Unidad. Cincuenta y cinco Unidades $(80 \%)$ carecen de anestesistas específicos. El número de pancreatectomías realizadas durante 2017 en los centros encuestados fue 1.315 duodenopancreatectomías cefálicas (DPC), 566 pancreatectomías distales (PD) y 178 duodenopancreatectomías totales (DPT). La media por centro fueron 19,1 DPC, 8,2 PD y 2,6 DPT. La DPC más habitual se realiza mediante abordaje clásico, con pancreatoyeyunostomía, montaje en un asa, con gastroyeyunostomía antecólica y 2 drenajes. Solo 7 Unidades efectúan la DPC por laparoscopia y solamente 13 Unidades no realizan PD laparoscópica.

Conclusiones: Esta encuesta proporciona información actualizada del trabajo asistencial y científico de un gran porcentaje de las Unidades donde se realiza cirugía pancreática en España, y además puede servir de punto de partida a trabajos multicéntricos prospectivos.
\end{abstract}

(C) 2019 Publicado por Elsevier España, S.L.U. en nombre de AEC.

\footnotetext{
* Autor para correspondencia.

Correo electrónico: jose_ramia@hotmail.com (J.M. Ramia).

$\diamond$ Los nombres de los componentes del Grupo Español de Cirugía Pancreática (AEC/CE-IHPBA) están relacionados en el anexo. https://doi.org/10.1016/j.ciresp.2019.02.007

0009-739X/C 2019 Publicado por Elsevier España, S.L.U. en nombre de AEC.
} 
Keywords:

Survey

Pancreas

Technique

Surgery

Review

\section{National survey on Pancreatic Surgery Units}

\author{
A B S T R A C T
}

Introduction: The technical, human, scientific and treatment characteristics of the Units that manage complex pathologies have not been studied in depth.

Methods: Multi-institutional descriptive study (survey) developed jointly by the Hepatobiliary-Pancreatic Division of the Spanish Association of Surgeons and the Spanish Chapter of the IHPBA (International Hepatopancreatobiliary Association) on the characteristics of the Units where pancreatic surgery is performed in Spain.

Results: 82 surveys were sent. 69 medical centers responded (84\%), belonging to 16 autonomous regions of Spain. The total population of these regions was $23,183,262$ (50\% of the Spanish population). The average number of beds per hospital was 673 . The unit that performs pancreatic surgery is a Hepatobiliary-Pancreatic Surgery Unit or HPB and Liver Transplant Surgery Unit in 56 hospitals (77\%). The average number of surgeons is 4.5 per Unit. Fifty-five Units (80\%) lack specific anesthetists. The number of pancreatectomies performed during 2017 at the hospitals surveyed was 1,315 pancreaticoduodenectomies (PD), 566 distal pancreatectomies (DP) and 178 total pancreaticoduodenectomies (TPD). The mean per hospital was 19.1 PD, 8.2 DP and 2.6 TPD. PD was usually performed using a classic approach, with pancreatojejunostomy, single-loop technique, antecolic gastrojejunostomy and using two drain tubes. Only 7 Units performed PD laparoscopically and only 13 units did not perform laparoscopic DP.

Conclusions: This survey provides updated information about the majority of the Units where pancreatic surgery is performed in Spain and could also serve as a starting point for prospective multicenter studies.

(C) 2019 Published by Elsevier España, S.L.U. on behalf of AEC.

\section{Introducción}

La información existente sobre qué centros efectúan una determinada patología, qué volumen de enfermos afectos atienden, qué características humanas y estructurales tienen las Unidades existentes y las técnicas que desarrollan es muy escasa tanto a nivel nacional como internacional. La literatura sobre qué características deben cumplir un centro y una Unidad que realicen cirugía pancreática es muy escasa, y solo valdría la existente en cada país ya que los sistemas sanitarios son muy diferentes entre las diversas naciones, y las características exigidas en un determinado país no son extrapolables a otro ${ }^{1}$.

La sección Hepatobiliopancreática de la Asociación Española de Cirujanos y el capítulo español de la IHPBA (Asociación Internacional Hepatopancreatobiliar) decidieron efectuar conjuntamente una encuesta para identificar qué centros efectúan cirugía pancreática, qué características estructurales tenían dichos centros y Unidades, qué volumen de enfermos eran operados en cada centro, su producción científica y qué técnicas se realizaban, con el objetivo de obtener una información actualizada del estado de la cirugía pancreática en España que permita ser la base de posteriores trabajos multicéntricos similares a los realizados en otros países europeos.

\section{Métodos}

Estudio descriptivo multiinstitucional. La sección Hepatobiliopancreática de la Asociación Española de Cirujanos y el capítulo español de la IHPBA han desarrollado conjuntamente una encuesta informativa sobre las características de las Unidades donde se realiza cirugía pancreática. Se ha enviado dicha encuesta, realizada en Google Drive ${ }^{\mathrm{TM}}$, a todos los hospitales que participaron en el Registro sobre Cirugía Pancreática efectuado en 2015. Además, en el correo electrónico donde se presentaba la encuesta se incluía el listado de participantes inicial, y se solicitó que si se conocían otros centros que realizaran cirugía pancreática y no estuvieran incluidos en la primera lista se facilitara sus datos para intentar incluir la totalidad de centros nacionales que realizan cirugía pancreática.

La encuesta incluía 4 grupos de preguntas: datos generales del centro hospitalario, datos de la Unidad, datos sobre la persona que rellena la encuesta y datos técnicos (tabla 1).

\section{Resultados}

Se enviaron 82 encuestas. Respondieron 69 centros (84\% de las encuestas enviadas) pertenecientes a 16 comunidades autónomas (tabla 2). Con relación a los centros participantes, 66 son de titularidad pública y 3 son centros privados. Cuarenta y ocho centros son hospital de referencia para otras áreas de salud $(69,6 \%)$ y 21 solo atienden a pacientes de su área sanitaria.

La suma de habitantes de las áreas propias de los hospitales consultados es 23.183.262. Teniendo en cuenta que según los datos provisionales poblacionales del Instituto Nacional de Estadística de 2018 en España hay 46.659.302 habitantes, 
Tabla 1 - Encuesta de cirugía pancreática

Encuesta cirugía pancreática

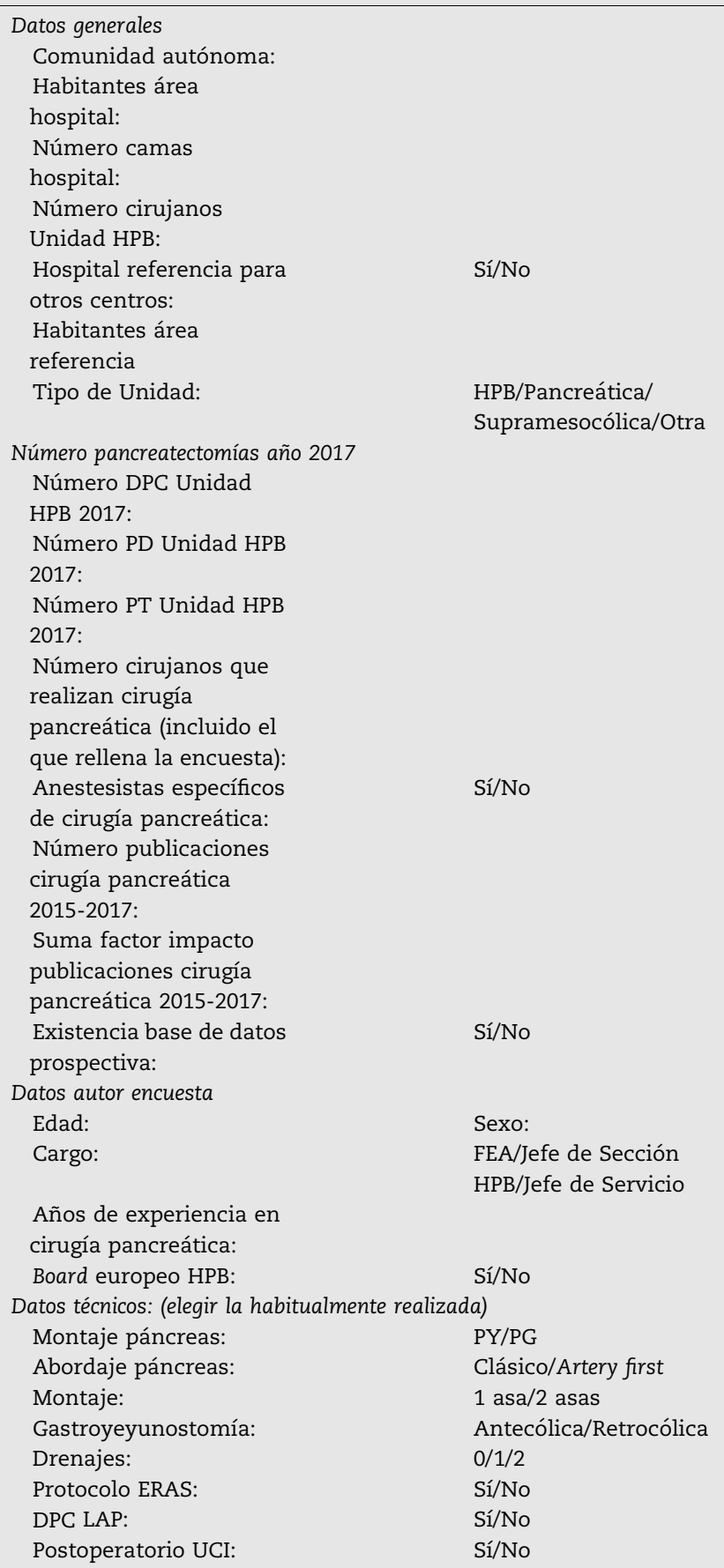

DPC: duodenopancreatectomía cefálica; ERAS: Enhanced Recovery After Surgery; FEA: facultativo especialista de área; HPB: hepatobiliopancreática; LAP: laparoscópica; PD: pancreatectomía distal; PG: pancreatogastrostomía; PT: pancreatectomía total; PY: pancreatoyeyunostomía; UCI: Unidad de Cuidado Intensivo.

toyeyunostomia; UCI: Unidad de Cuidado Intensivo.

supone el 49,7\% de la población total de España. El número de habitantes atendidos como hospital de referencia es 21.607 .524 (46,31\%). Por tanto, sumando el área propia de todos los hospitales participantes y el área atendida como
Tabla 2 - Distribución de los centros por comunidades autónomas

\begin{tabular}{ll} 
Comunidad autónoma & Número de hospitales \\
\hline Andalucía & 10 \\
Aragón & 3 \\
Asturias & 1 \\
Baleares & 1 \\
Canarias & 2 \\
Cantabria & 1 \\
Castilla-La Mancha & 6 \\
Castilla y León & 4 \\
Cataluña & 9 \\
Euskadi & 5 \\
Extremadura & 1 \\
Galicia & 1 \\
Madrid & 13 \\
Murcia & 1 \\
Navarra & 2 \\
Comunidad Valenciana & 9 \\
\hline
\end{tabular}

hospital de referencia $(23.183 .262+21.607 .524)$ son un total de 44.790.786 habitantes, lo que supone un $96 \%$ de la población española.

El número medio de camas de los hospitales es 673 (rango: 150-1403). La distribución por número de camas es la siguiente, 0-500 camas: 25 (36\%); 501-1.000 camas: 37 (54\%), y $>1.000$ camas: 7 (10\%). La Unidad que realiza la cirugía pancreática es una Unidad de Cirugía Hepatobiliopancreática o Cirugía HPB y Trasplante Hepático en 56 hospitales (77\%), en 4 de cirugía supramesocólica, en 4 de cirugía biliopancreática, exclusivamente de páncreas en un centro, y otras denominaciones en 4 hospitales. El número medio de cirujanos es 4,5 por cada Unidad (rango: 1-11) y la mediana es 4 . Cincuenta y cinco Unidades $(80 \%)$ carecen de anestesistas específicos para la Unidad que realiza la cirugía pancreática.

En el trienio 2015-2017, 37 Unidades no publicaron ningún artículo (53,6\%) y 32 Unidades, un total de 145 con un factor de impacto de 272,515 . La media de publicaciones en los centros publicadores es de 4,53 por Unidad y la mediana es 3 . El factor de impacto medio por publicación es 1,88 . Cincuenta y cinco Unidades $(80 \%)$ tienen una base de datos prospectiva.

El número de pancreatectomías realizadas durante 2017 en los centros encuestados se describe en la tabla 3. Sobre las preguntas técnicas, el abordaje habitual es el denominado clásico en 44 Unidades (64\%), artery first en 9, y ambos en 16.

Tabla 3 - Número de pancreatectomías en 2017

\begin{tabular}{llll} 
Número & \multicolumn{1}{c}{ DPC } & \multicolumn{1}{c}{ PD } & \multicolumn{1}{c}{ DPT } \\
\hline Total & 1.315 & 566 & 178 \\
Media & $19,1(3-54)$ & $8,2(0-33)$ & $2,6(0-24)$ \\
$<10$ & $19(27,5 \%)$ & $50(72,5 \%)$ & $66(95,6 \%)$ \\
$11-20$ & $25(35,4 \%)$ & $16(23,2 \%)$ & $3(4,3 \%)$ \\
$21-40$ & $20(29 \%)$ & $3(4,3 \%)$ & \\
$>40$ & $5(7,2 \%)$ & & \\
\hline
\end{tabular}

DPC: duodenopancreatectomía cefálica; DPT: duodenopancreatec tomía total; PD: pancreatectomía distal. 
Tabla 4 - Características del cirujano que rellenó la encuesta

\begin{tabular}{lllll} 
& \multicolumn{1}{c}{$\mathrm{N}$} & \multicolumn{1}{c}{ Edad } & Sexo (M/H) & Años CP \\
\hline FEA & $23(33,3 \%)$ & $36(34-59)$ & $7 / 16$ & $10,9(4-30)$ \\
Jefe de Sección & $29(42 \%)$ & $55,6(46-63)$ & $0 / 29$ & $21,7(6-35)$ \\
Jefe de Servicio & $17(24,7 \%)$ & $58,3(44-68)$ & $3 / 14$ & $24,2(6-30)$ \\
Total & 69 & $49,7(34-68)$ & $10 / 59$ & $18,7(4-35)$ \\
\hline
\end{tabular}

CP: cirugía pancreática; FEA: facultativo especialista de área; $H$ : hombre; M: mujer.

La anastomosis pancreatoyeyunal habitual que realizan es la pancreatoyeyunostomía en 54 Unidades (78\%), la pancreatogastrostomía (PG) en 10 Unidades y ambas sin predilección en 5 Unidades. En el montaje se emplea una sola asa en 39 Unidades (56\%), 2 asas en 28 Unidades y ambas técnicas en 2. La anastomosis gastroyeyunal habitual está situada antecólica en 51 (74\%) y retrocólica en 18. Tres Unidades no colocan ningún drenaje, en 15 Unidades un único drenaje y 2 drenajes en 51 Unidades.

No existe protocolo de rehabilitación multimodal en 45 Unidades $(64,2 \%)$ y 24 sí lo tienen. Solo 7 Unidades efectúan la duodenopancreatectomía cefálica (DPC) por laparoscopia y 62 Unidades $(89,9 \%)$ no la realizan. Trece unidades no realizan pancreatectomía distal (PD) por abordaje laparoscópico, y 56 sí la realizan (81,2\%). El postoperatorio inmediato de la DPC se realiza en la UCI en 53 hospitales y en 16 no en la UCI sino en la Reanimación anestésica.

Las características del cirujano que ha rellenado la encuesta (edad, sexo, cargo y experiencia en cirugía pancreática) están incluidas en la tabla 4.

\section{Discusión}

Esta es la primera encuesta sobre la actividad asistencial y científica de un gran porcentaje de las Unidades donde se realiza cirugía pancreática en España.

Las características de estas Unidades son: un 53,7\% de los hospitales donde se encuentran estas Unidades en las que se realiza cirugía pancreática son de tamaño medio y alto (500 a 1.000 camas), pero un $36 \%$ se realiza en hospitales con menos de 500 camas. Las Unidades en la mayoría de las ocasiones (77\%) atienden a los pacientes que precisan cirugía hepática, biliar y pancreática. El número medio de cirujanos es de 4,5, con una amplia variabilidad que oscila entre uno y 11 cirujanos, lo que lógicamente afecta a las pancreatectomías/cirujano por año. El $80 \%$ de los centros carecen de anestesistas específicos para cirugía pancreática, dato muy importante ya que Uppal et al. han demostrado en un estudio de 2018 que la existencia de un grupo de anestesistas especializados en este tipo de cirugías mejora los resultados obtenidos, y sería un objetivo en los grandes hospitales con amplias plantillas de anestesistas ${ }^{2}$. Casi el $80 \%$ de pacientes son remitidos a la UCI durante el postoperatorio inmediato.

Con respecto a la producción científica, creemos que los datos reales son inferiores a los obtenidos, ya que existen estudios multicéntricos nacionales que hacen que hayan artículos duplicados en la cuenta final de artículos. El 31 de diciembre de 2018 realizamos una búsqueda en Pubmed 20152017 (con las palabras pancreas AND surgery AND Spain) y solo obtuvimos 32 resultados.

En la década de los 90 se publican artículos muy controvertidos sobre el beneficio de la regionalización de procedimientos en los que se afirma que hay relación directa entre el volumen de enfermos intervenidos de una determinada patología compleja y los resultados obtenidos, especialmente en lo referente a complicaciones postoperatorias, mortalidad, estancia media y fallo al rescate cuando existen complicaciones $^{3,4}$. Existen también publicaciones contradictorias entre la relación volumen de casos y costes ${ }^{3,4}$.

Se han empleado diferentes valores de corte para definir centros de bajo, medio y alto volumen de cirugía pancreática $^{3,5}$. El estudio holandés de Van der Geest et al. define como unidad de alto volumen aquellas que realizan más de 40 DPC, observando que en estos centros la mortalidad es inferior y la supervivencia es superior, datos probablemente relacionados con que estos centros obtienen un mayor número de ganglios extirpados, mayor tasa de RO y de quimioterapia postoperatoria ${ }^{5}$. Estos mejores resultados son especialmente llamativos en los pacientes ancianos ${ }^{6}$. En España solo 5 centros realizan más de 40 DPC/año, lo que supone un 7,2\% del total de las DPC realizadas. También solo 5 centros en Holanda realizan más de 40 DPC/año, pero suponen el 36\% del total de DPC realizadas, lo que implica una regionalización de procedimientos muy superior a la de nuestro país. La media de DPC en Holanda por centro es de 23 DPC, ligeramente superior a las 19 DPC obtenidas en nuestra encuesta. La información sobre PD y duodenopancreatectomías totales (DPT) en relación con el volumen anual es muy limitada en la literatura. Solo 3

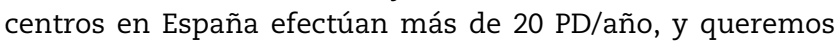
destacar la amplia variabilidad en el empleo de la DPT por centros que probablemente indique una diferente indicación de esta intervención, como la DPT en casos de alto riesgo de fallo anastomótico no aceptada por todos los cirujanos pancreáticos ${ }^{7}$.

En 2002, Figueras et al. propusieron que para ser hospital de referencia se deberán realizar al menos 24 DPC/año; no hemos encontrado datos en otros países sobre este punto ${ }^{1}$. Otro tema también muy debatido es el número mínimo de pancreatectomías por año que debe realizar cada centro y cada cirujano, no existiendo en la actualidad un consenso sobre qué número de corte es el adecuado ${ }^{5}$.

Si queremos definir cómo es la DPC más frecuente que se realiza en España, podríamos decir que es efectuada mediante laparotomía realizando un abordaje clásico, con anastomosis pancreatoyeyunal, montaje en un asa, gastroyeyunostomía antecólica y empleo de drenajes intraabdominales. Vamos a profundizar en estos aspectos brevemente, comprobando que empleando los metaanálisis publicados más recientes, en la mayoría de las opciones técnicas quedan muchas dudas por resolver ya que la superioridad de una opción frente a otra no suele ser evidente, y habitualmente el cirujano tiende a emplear la técnica que cree mejor o con la que está familiarizado.

El abordaje conocido como artery first se ha popularizado recientemente en la cirugía pancreática, ya que permite teóricamente comprobar la resecabilidad del tumor pancreá- 
tico y permite obtener mayores resecciones R0. El metaanálisis de Ironside et al. demuestra que este abordaje se acompaña de menor pérdida sanguínea intraoperatoria, menor transfusión intraoperatoria, menor morbilidad, menor incidencia de fístula grado B/C, mayor tasa de resecciones R0, mejor supervivencia y una mortalidad similar a la técnica tradicio$\mathrm{nal}^{8}$. Pese a ser una modificación técnica bastante reciente, un tercio de las Unidades ya la practica de forma sistemática o en combinación con el abordaje clásico.

La fístula de la anastomosis pancreatodigestiva es la complicación más grave tras una DPC. Muchas técnicas alternativas se han realizado intentando mejorar la ya clásica anastomosis pancreatoyeyunal ductomucosa. Entre las opciones alternativas destaca la $\mathrm{PG}^{9}$. En el estudio aleatorizado realizado en España por Figueras et al., comparando anastomosis pancreatoyeyunal y PG, demostraron que la tasa y severidad de la fístula pancreática era inferior con la $\mathrm{PG}^{9}$. El metaanálisis de Menahem et al. confirmó dichos resultados ${ }^{10}$. Pese a estos excelentes datos, la PG sigue siendo una técnica minoritariamente empleada en España: el 80\% de las Unidades emplean la anastomosis pancreatoyeyunal y en un $20 \%$ se emplea la PG únicamente o ambas técnicas. No hay datos internacionales que permitan comparar estas cifras con las obtenidas en España.

El posicionamiento de la anastomosis gastroyeyunal tras DPC puede ser antecólico o retrocólico. Los que preconizan la posición antecólica defienden que si se sitúa en esta posición se aleja de la anastomosis pancreatoyeyunal, y si se produce una fístula pancreática, la anastomosis gastroyeyunal no se ve comprometida y permite mantener el tránsito digestivo oral. En el metaanálisis de Joliat et al. la gastroyeyunostomía antecólica no mejora los resultados cuando se compara con la realizada por vía retrocólica ${ }^{11}$. En España, se utiliza mayoritariamente la gastroyeyunostomía antecólica. Respecto al montaje en un asa o 2 asas, el metaanálisis de Deng et al. concluye que ambas técnicas obtienen resultados similares en cuanto a complicaciones postoperatorias ${ }^{12}$; y a nivel nacional, el montaje en un asa es levemente más empleado que el realizado en 2 asas.

El uso de drenajes en cirugía pancreática es un tema controvertido. Un estudio aleatorizado comparando no drenaje vs. drenaje en DPC fue suspendido al comprobar que el grupo sin drenaje tenía una mortalidad superior (3\% vs. $12 \%)^{13}$. Huan et al. han realizado posteriormente un metaanálisis concluyendo que la tasa de fístula pancreática postoperatoria es inferior en el grupo sin drenaje que en el grupo de drenaje rutinario, poner drenajes incrementa la morbilidad tras DPC, y no usar drenaje en PD reduce la mortalidad pero la tasa de fístula clínicamente relevante es superior $^{14}$. La conclusión del metaanálisis es que no debería usarse drenaje en la PD, y no rutinariamente en la DPC ${ }^{14}$. La política de no drenajes está muy poco extendida en España y solo 3 Unidades la practican, la mayoría de las cuales usan 2 drenajes abdominales.

La cirugía pancreática laparoscópica ha tardado en difundirse dentro de las Unidades de Cirugía Pancreática, especialmente la DPC ya que es técnicamente muy demandante ${ }^{15,16}$. En una encuesta mundial sobre cirugía pancreática laparoscópica realizada entre cirujanos pancreáticos, el 79\% de ellos realizaban PD laparoscópica y un 29\% DPC laparoscópica. El 70\% pensaba que la PD laparoscópica es superior a la abierta, pero solo un $10 \%$ cuando se refería a la DPC laparoscópica. Pese a estos datos, la PD laparoscópica probablemente no está tan difundida como parece. En 2015, el Grupo Holandés de Cáncer de Páncreas (Dutch Cancer Study Group) publicó que en el periodo 2005-2013, solo el 10\% de las PD en Holanda se habían realizado por abordaje laparoscópico; tras ello se implantó un sistema formativo tutorizado nacional que ha incrementado su utilización. En un estudio mundial, recién publicado, comparando DPC laparoscópica y DPC abierta, solo el $17 \%$ de los pacientes se habían intervenido por abordaje laparoscópico, robótico o híbrido ${ }^{17}$. Los datos de nuestra encuesta son superiores a los mencionados previamente, ya que en $\mathrm{PD}$ el $90 \%$ de los grupos realizan PD laparoscópica, aunque no se ha podido precisar el porcentaje exacto de PD realizadas por laparoscopia, y únicamente un $10 \%$ de los grupos realizan DPC laparoscópica. Solo un registro nacional de todas las pancreatectomías realizadas en España podrá permitir obtener una información fiable. La implantación de la DPC laparoscópica probablemente sea un proceso aún muy lento.

Los protocolos de rehabilitación multimodal han demostrado que disminuyen la estancia y los costes sin incrementar la morbimortalidad; casi un tercio de las Unidades tienen estos protocolos para el postoperatorio de las pancreatectomías. Si bien estos protocolos tienen diversos niveles de complejidad según el número de actuaciones que se realicen, creemos que es una tasa muy relevante teniendo en cuenta que las pancreatectomías, por su baja frecuencia y complejidad, no suelen ser el grupo donde se inicia el empleo de la rehabilitación multimodal ${ }^{18}$.

Una de las limitaciones de esta encuesta es que no recoge la totalidad de Unidades, los datos no son prospectivos y no han sido auditados por un auditor externo- La fortaleza del estudio es que la suma de las áreas hospitalarias propias y de referencia alcanza un 95\% del territorio nacional, contando con la participación de centros de 16 comunidades autónomas; y además hemos incluido no solo aquellos centros de titularidad pública, sino también 3 centros privados.

Como conclusión, creemos que esta encuesta proporciona una información actualizada del trabajo asistencial y científico de un gran porcentaje de las Unidades donde se realiza cirugía pancreática en España, y además puede servir de punto de partida a trabajos multicéntricos prospectivos. Creemos que, al igual que en otros países como Holanda, o en otras patologías como el cáncer gástrico, futuros registros prospectivos que incluyan la totalidad de las pancreatectomías realizadas pueden ofrecer una información veraz sobre los resultados quirúrgicos y oncológicos en una patología especialmente compleja como es la cirugía del páncreas.

\section{Conflicto de intereses}

Los autores declaran no tener ningún conflicto de intereses. 


\section{Anexo. Grupo Español de Cirugía Pancreática (AEC/CE-IHPBA)}

Los componentes del grupo se presentan por orden alfabético (comunidad autónoma y cirujano).

\begin{tabular}{|c|c|c|c|}
\hline Comunidad autónoma & Participantes & Hospital & Ciudad \\
\hline \multirow[t]{10}{*}{ Andalucía } & D. Bejarano & H. J.R. Jiménez & Huelva \\
\hline & A. Calvo & H. Puerto Real & Cádiz \\
\hline & M.J. Castro & H. Puerta del Mar & Cádiz \\
\hline & E. Daban & H. San Cecilio & Granada \\
\hline & M.A. Gomez-Bravo & H. Virgen del Rocío & Sevilla \\
\hline & P. Parra & H. Valme & Sevilla \\
\hline & J. Santoyo & H. Carlos Haya & Málaga \\
\hline & M. A. Suarez & H. Virgen Victoria & Málaga \\
\hline & T. Villegas & H. Virgen de las Nieves & Granada \\
\hline & L. Tejedor & H. Algeciras & Algeciras \\
\hline \multirow[t]{3}{*}{ Aragón } & J.L. Blas & H. Royo Vilanova & Zaragoza \\
\hline & A. Garcia-Gil & H. Clínico Lozano Blesa & Zaragoza \\
\hline & M. Serradilla & H. Miguel Servet & Zaragoza \\
\hline Asturias & A. Miyar & H. Central de Asturias & Oviedo \\
\hline Baleares & R. Morales & H. Son Espases & Palma de Mallorca \\
\hline \multirow[t]{2}{*}{ Canarias } & J. Larrea & H. Insular Gran Canaria & Las Palmas \\
\hline & P. Sanz & H. N.S. Candelaria & Tenerife \\
\hline Cantabria & J.C. Rodriguez Sanjuan & H. M. Valdecilla & Santander \\
\hline \multirow[t]{6}{*}{ Castilla-La Mancha } & R. de Miguel & H. Virgen de la Luz & Cuenca \\
\hline & S. Martinez & H. N. S. Prado & Talavera \\
\hline & J.I. Miota & C. H. Albacete & Albacete \\
\hline & D. Padilla & H. Ciudad Real & Ciudad Real \\
\hline & J. M. Ramia & H. Guadalajara & Guadalajara \\
\hline & P. Toral & H. Virgen de la Salud & Toledo \\
\hline \multirow[t]{4}{*}{ Castilla y León } & J. Beltran & H. Clínico Valladolid & Valladolid \\
\hline & B. Ielpo & H. León & León \\
\hline & L. Muñoz & H. Clínico Salamanca & Salamanca \\
\hline & D. Pacheco & H. Río Hortega & Valladolid \\
\hline \multirow[t]{9}{*}{ Cataluña } & J.M. Badia & H. Granollers & Granollers \\
\hline & E. Cugat & H. Terrassa/Germans Trias i Pujol & Terrassa/Barcelona \\
\hline & A. Escartin & H. Arnau de Vilanova & Lleida \\
\hline & L. Falgueras & H. Josep Trueta & Girona \\
\hline & J. Fabregat & H. Bellvitge & Barcelona \\
\hline & F. Garcia-Borobia & H. Parc Taulí & Sabadell \\
\hline & R. Jorba & H. Joan XXIII & Tarragona \\
\hline & E. Pando & H. Vall d'Hebron & Barcelona \\
\hline & I. Poves & H. del Mar & Barcelona \\
\hline \multirow[t]{5}{*}{ Euskadi } & A. Valdivieso & H. Cruces & Bilbao \\
\hline & L. Garcia & H. Galdakao & Galdakao \\
\hline & I. Martinez & H. Araba & Vitoria \\
\hline & P. Gomez & H. Basurto & Basurto \\
\hline & M. Alkorta & H. Donostia & San Sebastián \\
\hline Extremadura & G. Blanco & H. Infanta Cristina & Badajoz \\
\hline Galicia & E. Varo & H. Clínico de Santiago & Santiago \\
\hline \multirow[t]{13}{*}{ Comunidad de Madrid } & J.M. Asencio & H. Gregorio Marañón & Madrid \\
\hline & A. Carabias & H. Getafe & Getafe \\
\hline & J. Castell & H. La Paz & Madrid \\
\hline & E. de Vicente & H. Sanchinarro & Madrid \\
\hline & L. Diez & H. Clínico Madrid & Madrid \\
\hline & T. Georgiev & Hospitales Grupo IDC & Madrid \\
\hline & A. Gutierrez & H. Príncipes de Asturias & Alcalá de Henares \\
\hline & V. Herrera & H. Infanta Cristina & Parla \\
\hline & C. Loinaz & H. 12 de Octubre & Madrid \\
\hline & E. Martin & H. La Princesa & Madrid \\
\hline & F. Ochando & F. Alcorcón & Alcorcón \\
\hline & F. Pereira & H. de Fuenlabrada & Fuenlabrada \\
\hline & A. Sanjuanbenito & H. Ramón y Cajal & Madrid \\
\hline & F. Sanchez-Bueno & H. Virgen Arrixaca & Murcia \\
\hline \multirow[t]{2}{*}{ Comunidad Foral de Navarra } & J. Herrera & C. Hosp. Navarra & Pamplona \\
\hline & F. Pardo & Clínica Univ. Navarra & Pamplona \\
\hline
\end{tabular}


Anexo. (Continuación)

Comunidad autónoma

Comunidad Valenciana

Participantes

J. Aguilo

E. Artigues

F. Asencio

J. A. Barreras

C. Domingo

R. Estevan

R. Lopez-Andujar

F. Lluis

A. Moya

L. Sabater

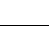

\section{B I B L I O G R A F Í A}

1. Figueras J, Fabregat J, Jaurrieta E, Valls C, Serrano T. Equipamiento, experiencia mínima y estándares en la cirugía hepatobiliopancreática (HBP). Cir Esp. 2002;71:171217.

2. Uppal A, Vuong B, Dehal A, Stern SL, Mejia J, Weerasinghe R, et al. Can high-volume teams of anesthesiologists and surgeons decrease perioperative costs for pancreatic surgery? HPB (Oxford). 2018;34447-52. http://dx.doi.org/ 10.1016/j.hpb.2018.09.008 [En prensa].

3. Sosa JA, Bowman HM, Gordon TA, Bass EB, Yeo CJ, Lillemoe $\mathrm{KD}$, et al. Importance of hospital volume in the overall management of pancreatic cancer. Ann Surg. 1998;228:42938.

4. Gani F, Johnston FM, Nelson-Williams H, Cerullo M, Dillhoff ME, Schmidt CR, et al. Hospital volume and the costs associated with surgery for pancreatic cancer. J Gastrointest Surg. 2017;21:1411-9.

5. Van der Geest LGM, van Rijssen LB, Molenaar IQ de Hingh IH, Koerkamp BG, Busch ORC, et al. Volume outcome relationships in pancreatoduodenectomy for cancer HPB. HPB (Oxford). 2016;18:317-24.

6. Van der Geest LG, Besselink MG, Busch OR, de Hingh IH, van Eijck CH, Dejong CH, et al. Elderly patients strongly benefit from centralization of pancreatic cancer surgery: a population-based study. Ann Surg Oncol. 2016;23:2002-9.

7. Eshmuminov D, Schneider MA, Tschuor C, Raptis DA, Kambakamba P, Muller X, et al. Systematic review and meta-analysis of postoperative pancreatic fistula rates using the updated 2016 International Study Group Pancreatic Fistula definition in patients undergoing pancreatic resection with soft and hard pancreatic texture. HPB (Oxford). 2018;20:992-1003.

8. Ironside N, Barreto SG, Loveday B, Shrikhande SV, Windsor JA, Pandanaboyana S. Meta-analysis of an artery-first approach versus standard pancreatoduodenectomy on perioperative outcomes and survival. Br J Surg. 2018;105:628-36.

9. Figueras J, Sabater L, Planellas P, Muñoz-Forner E, Lopez-Ben $\mathrm{S}$, Falgueras L, et al. Randomized clinical trial of pancreaticogastrostomy versus pancreaticojejunostomy on the rate and severity of pancreatic fistula after pancreaticoduodenectomy. Br J Surg. 2013;100:1597-605.

10. Menahem B, Guittet L, Mulliri A, Alves A, Lubrano J. Pancreaticogastrostomy is superior to pancreaticojejunostomy for prevention of pancreatic fistula after pancreaticoduodenectomy: an updated meta-analysis of randomized controlled trials. Ann Surg. 2015;261:882-7.

11. Joliat GR, Labgaa I, Demartines N, Schäfer M, Allemann P. Effect of antecolic versus retrocolic gastroenteric reconstruction after pancreaticoduodenectomy on delayed gastric emptying: a meta-analysis of six randomized controlled trials. Dig Surg. 2016;33:15-25.

12. Deng LH, Xiong JJ, Xia Q. Isolated Roux-en-Y pancreaticojejunostomy versus conventional pancreaticojejunostomy after pancreaticoduodenectomy: a systematic review and meta-analysis. J Evid Based Med. 2017;10:37-45.

13. Van Buren G, Bloomston M, Hughes SJ, Winter J, Behrman $\mathrm{SW}$, Zyromski NJ, et al. A randomized prospective multicenter trial of pancreaticoduodenectomy with and without routine intraperitoneal drainage. Ann Surg. 2014;259:605-12.

14. Huan L, Fei Q, Lin H, Wan L, Li Y. Is peritoneal drainage essential after pancreatic surgery? A meta-analysis and systematic review. Medicine (Baltimore). 2017;96:e9245.

15. Gurusamy KS, Riviere D, van Laarhoven CJH, Besselink M, Abu-Hilal M, Davidson BR, et al. Cost-effectiveness of laparoscopic versus open distal pancreatectomy for pancreatic cancer. PLoS One. 2017;12:e0189631.

16. De Rooij T, van Hilst J, van Santvoort H, Boerma D, van den Boezem P, Daams F, et al. Minimally invasive versus open distal pancreatectomy (LEOPARD): a multicenter patientblinded randomized controlled trial. Ann Surg. 2019;269:2-9.

17. Klompmaker S, van Hilst J, Wellner UF, Busch OR, Coratti A, D'Hondt M, et al. Outcomes after minimally-invasive versus open pancreatoduodenectomy: A Pan-European propensity score matched study. Ann Surg. 2018. http://dx.doi.org/ 10.1097/SLA.0000000000002850 [En prensa].

18. Pecorelli N, Nobile S, Partelli S, Cardinali L, Crippa S, Balzano $\mathrm{G}$, et al. Enhanced recovery pathways in pancreatic surgery: State of the art. World J Gastroenterol. 2016;22:6456-68. 